\title{
Permanent Pacemaker Implantation Through Persistent Left Superior Vena Cava: Safe for Normal Heart Position and Dextrocardia
}

\author{
Erika Maharani, Yoga Yuniadi
}

$\mathrm{P}$ ermanent pacemaker implantation in patient with persistent left superior vena cava (LSVC) has been reported in literature. Some authors has also been reported pacemaker implantation in patient with dextrocardia. Both of this situation has a challenging situation in placing the lead in right ventricle and risks of lead dislocation. We report two cases of successful pacemakers implantation,one with mirror image dextrocardia, in patient with persistent LSVC.

\section{Case I}

A 9-year-old female child, weighing 20 kilogram, was admitted to the hospital for ventricle septal defect (VSD) clossure. Echocardiography revealed dextrocardia with right sided apex,a perimembranous VSD with tricuspid regurgitation. During operation procedure the surgeon found bilateral superior vena cavawith the present ofinnominate vein. Right SVC was small and left SVC was big and drained into coronary sinus. Small left IVC went to coronary sinus and right IVC goes to the middle of right atrium.

\footnotetext{
Alamat Korespondensi:

Dr. dr. Yoga Yuniadi, SpJP. Divisi Aritmia, Departemen Kardiologi dan Kedokteran Vaskular, FKUI dan Pusat Jantung Nasional Harapan Kita. Jln S Parman Kav 87 Jakarta I I420. E-mail: yogay 136@gmail.com
}

Right and left ventricle were side by side. Clossure of the VSD using patch and repair of the tricuspid valve was done. Complete heart block with junctional escape rhythm (rate of $55 \mathrm{bpm}$ ) was occurred during the operation. Epicardial pacemaker was set with rate of 80 beat per minutes. Permanent pacing was indicated because of persistent atrioventricular block despite administration of methylprednisolon for 2 weeks.

Patient was transferred to the catheterization lab for pacemaker implantation. Under sterile condition and after appropiate sedation an $3 \mathrm{~cm}$ incision was made below the left clavicula after puncture of the left subclavian vein. The LSVC was accessed with no difficulty. A guidewire was advance into the vein and went to the coronary sinus and the right atrium. A52 $\mathrm{cm}$ active fixation lead (Medtronic 5076-52) was introduced to the vein through a $7 \mathrm{~F}$ peel away sheath. Active lead was preferred in this patient to prevent acute lead dislodgement.Hand-shaped stillet was succced in placing the lead in the right ventricle outflow tract (RVOT) using frontal and lateral projection. Early after implantation a pacing treshold of $0.6 \mathrm{~V}$ at $0.4 \mathrm{~ms}$, $\mathrm{R}$ wave amplitude of 7.2 $\mathrm{mV}$, impedance of $478 \mathrm{ohm}$, and current of $1 \mathrm{~mA}$ were obtained. Lead position in RVOT was confirmed using right anterior oblique (RAO) $30^{\circ}$ and left anterior oblique (LAO) $45^{\circ}$. The lead was connected to the single chamber generator (Medtronic Relia 


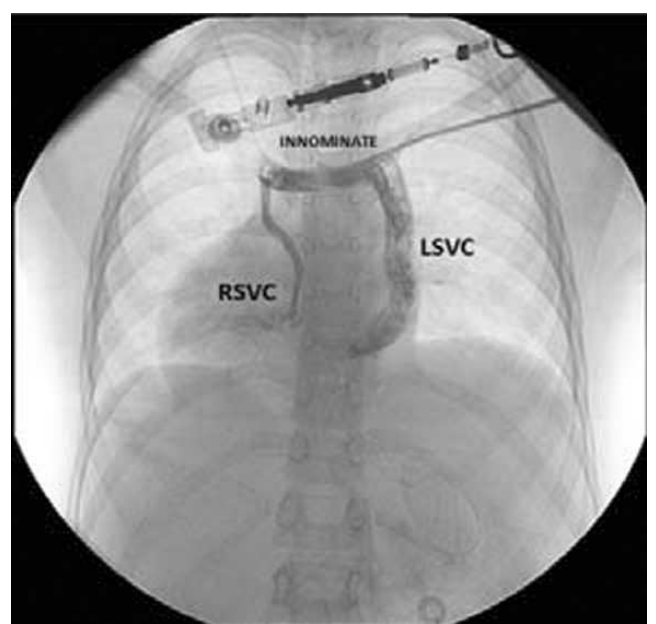

Figure 1. Venography of left subclavian vein persistent left superior vena cava. It drains to coronary sinus. Right superior vena cava was smaller and connect to LSVC trough innominate vein.
NWV613361S) and placed in the left subclavian pocket. Permanent pacemaker was set in VVIR with lower rate of 80 beat per minutes and upper rate of 140 beat per minutes. After an uneventful recovery the patient was discharge 3 days later. ECG of post PPM implantation was shown at figure 3.

\section{Case 2}

A 5-year-old female child, weighing 11 kilogram, developed complete atrioventricular block after underwent VSD clossure. The rhythm was not return to normal after two weeks observation, so permanent pacemaker implantation was planned. The patients was transferred to the catheterization lab. After appropiate sedation the right subclavian vein puncture was done. The peel away wire couldnot be advanced into the right
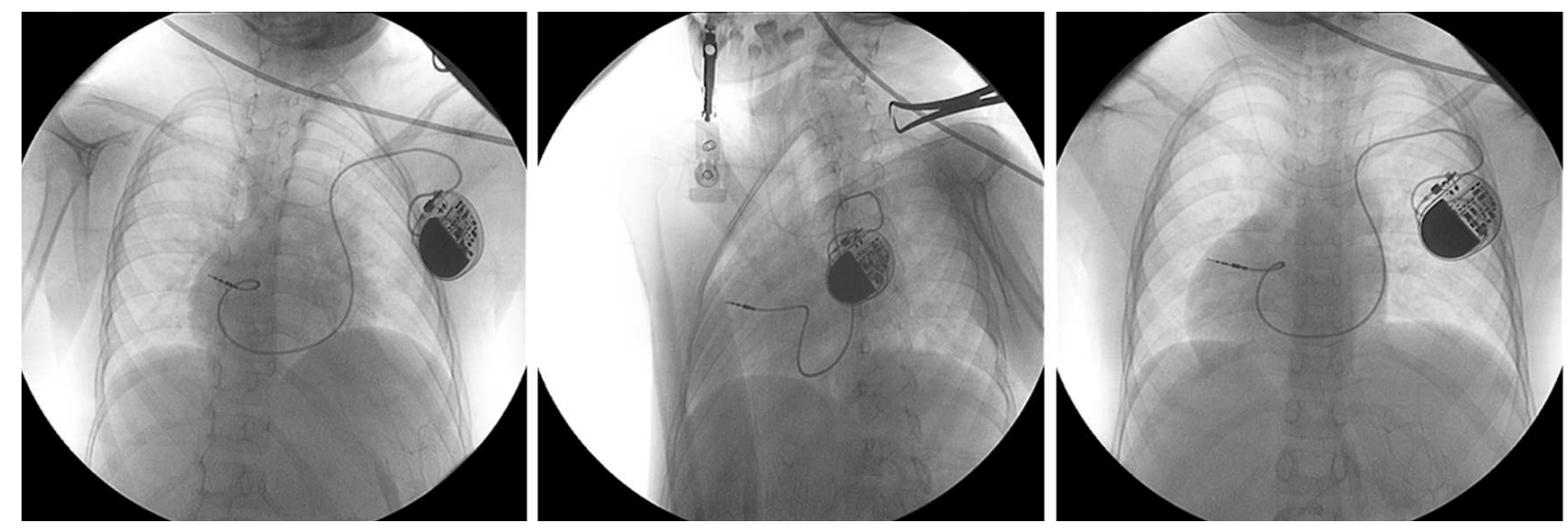

Figure 2. Active lead position in right ventricular outflow track (RVOT) from RAO 30o view (left panel), LAO 450 view (mid panel), and anteroposterior view (right panel).

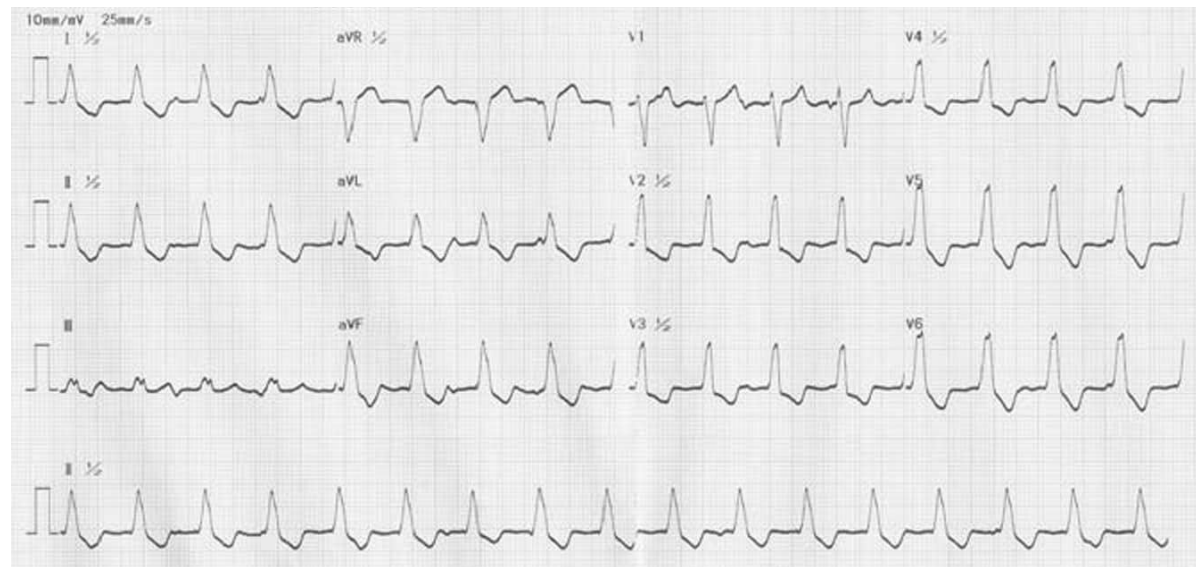

Figure 3. Twelve leads electrocardiogram after permanent pacemaker implantation. 


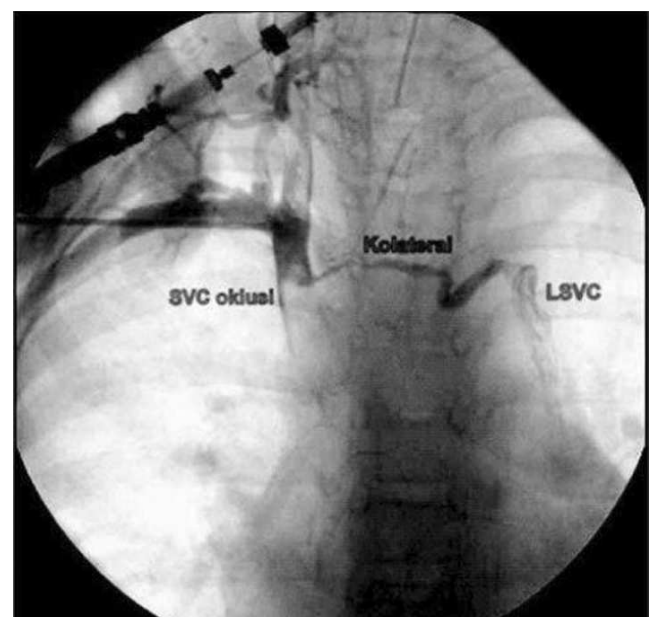

Figure 4. Venography of right subclavian vein showed occlussion of right superior vena cava. Note that there is a innominate vein that connect to persistent left superior vena cava. LSVC drains to coronary sinus which has a big diameter. atrium. Venography was performed and showed total oclussion of right superior vena cava (RSVC) due to trombus and contrast filled the persistent LSVC that drained to coronary sinus.

Access for permanent pacemaker implantation was decided through the persistent LSVC. About 3 $\mathrm{cm}$ incision below the left clavicula was done after puncture puncture of the left subclavian vein. A 7F peel away was inserted through the wire that went to coronary sinus and the right atrium. An $52 \mathrm{~cm}$ active lead can be placed in right ventricular outflow track (RVOT) using a hand-shaped stillet (with large loop) without any difficulty. The capture threshold measured $0.4 \mathrm{~V}$ at $0.4 \mathrm{~ms}$, $\mathrm{R}$ wave amplitude of ... $\mathrm{mV}$, with an impedance of $1399 \mathrm{ohm}$ and current of $0.6 \mathrm{~mA}$. Lead position in RVOT was confirmed using right anterior oblique of $30^{\circ}$ and left anterior oblique of $45^{\circ}$. Distal of the lead was located in basal septal of RVOT. Patient was discharge 5 days after the procedure.
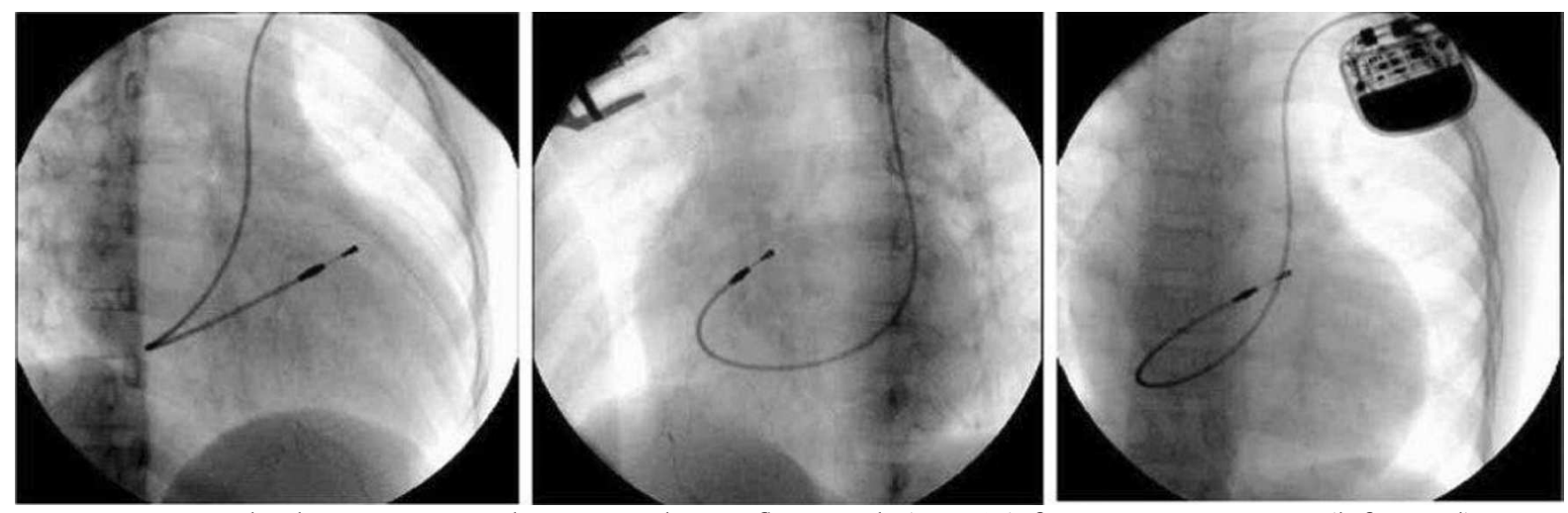

Figure 5. Active lead position in right ventricular outflow track (RVOT) from RAO 30o view (left panel), LAO 450 view (mid panel), and anteroposterior view (right panel)

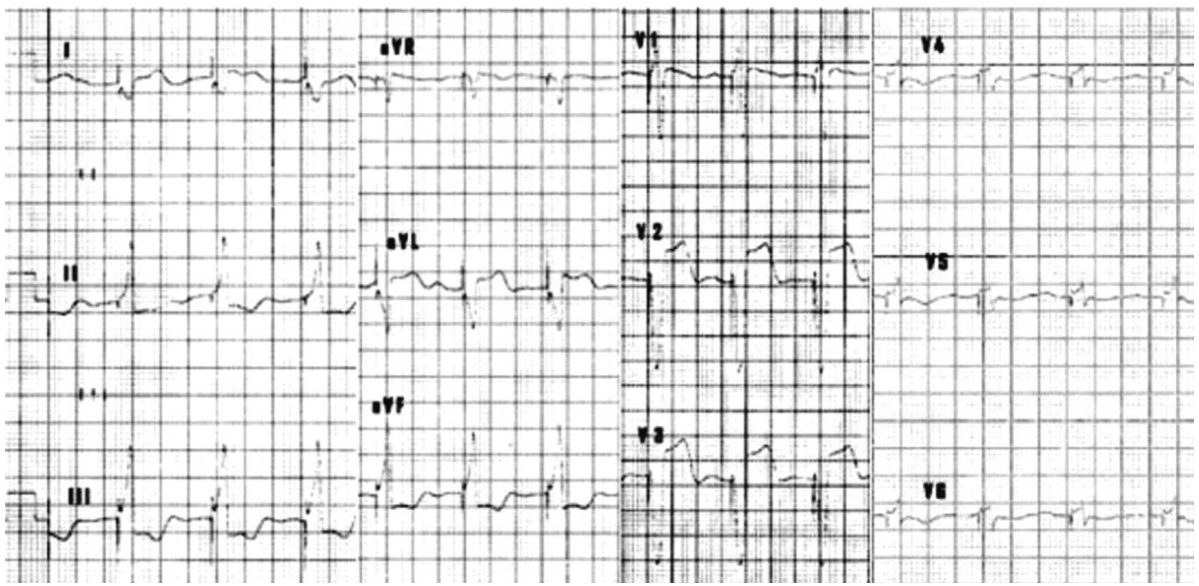

Figure 6. Twelve leads electrocardiogram after permanent pacemaker implantation 
Jurnal Kardiologi Indonesia

\section{Discussion}

\section{Complete heart block post surgery}

Approximately $1 / 3$ of all the patients after open heart surgery for congenital heart disease experienced cardiac arrhythmia during the early post-operative period. The incidence of complete heart block has decreased from $>10 \%$ in the 1960 s to $1-3 \%$ in the currentera. ${ }^{1}$ Nezafati observed post-operative atrioventricular (AV) block was occured in $7 \%$ patients, and $4 \%$ of them had a complete AV block. ${ }^{2}$ The surgical repairs most commonly associated with postoperative complete heart block are operations involving the interventricular septum, namely ventricular septal defect and atrioventricular canal defects. ${ }^{3}$ Edwin et al found that permanent post-operative complete heart block occurred in $1.3 \%$ of patients undergoing VSD repair. The dominant anatomic risk factor was a large perimembranous VSD as an isolated defect or as part of a conotruncal anomaly. The His bundle is intimately related to the posterior and inferior margins of the defect making it vulnerable to operative trauma. ${ }^{4}$ Nearly 10 $32 \%$ of patients with postoperative heart block have a recovered atrioventricular conduction. Batra in his data showed afterinsertion of the pacemaker, recovery of atrioventricular conductionwas recognized in 7 of 72 patients (9.6\%) at a median of 41days (18-113 days) after the initial cardiac operation. Pacing for acquired postoperative heart block should be considered after 9 days following cardiac surgery. ${ }^{1}$

\section{Pacemaker implantation in dextrocardia and persistent LSVC}

The incidence of dextrocardia is of 1 over 10,000 to 5 over 20,000 live births, when the heart is located in the right side of the hemithorax. In the majority of cases the dextrocardia is associated with cardiac malformations. Permanent endocardial pacing may deal with some difficulty, especially in positioning the lead in right ventricle and risks of lead dislocation, either in acute and chronic phase. ${ }^{5}$ Technical challenge of implanting pacemaker leads in these patients is the extra angle imposed on the leads, when passing through the abnormal course of superior vena cava and reversed position of right atrium and right ventricle. In patient with dilated heart further increases the difficulty of implanting lead. ${ }^{6}$ Angiographic investigation is important to perform a proper endocardial approach to verify the anatomic situation. ${ }^{5}$ The characteristic electrocardiographic findings inmirror-image dextrocardia are negative $P$ waves with inverted QRS complex and $T$ wave in lead 1 and the interchanging of lead aVL for aVR, of lead 2 for lead 3, and of right precordial leads for the corresponding left precordial leads. An upright $\mathrm{P}$ wave in lead 1 was frequently associated with either bilateral superior venae cavae or absent inferior vena cava. The $\mathrm{P}$-wave vector was thought to come from a left atrial rhythm in patient with bilateral superior venae cavae, while with absent inferior vena cava, there was thought to be a coronary sinus rhythm. ${ }^{7}$ Less than $10 \%$ of dextrocardia patients have associated congenital cardiac defects, but of those that do occur, anomalous venous return is common. Venous anomalies can arise in the form of abnormal pulmonary venous return or a persistent vena cava. ${ }^{8}$ Some dextrocardia patients presence with venous malformation, as the persistence of left superior vena cava draining in dilated coronary sinus. ${ }^{5}$

Left persistent superior vena cava (PSVC) is a congenital anomaly present in approximately 0.3 $0.5 \%$ of the general population. ${ }^{8,9}$ In the presence of congenital heart anomaly the prevalence is $10 \%$ higher. ${ }^{8,10}$ During cardiac development the left-sided anterior venous cardinal system disappears, leaving only the coronary sinus and a remnant known as ligament of Marshall. Failure of clossure of the left anterior cardinal vein results in a double caval system. ${ }^{10,11,12}$ In nearly $60 \%$ of these cases, there is an innominate vein which bridges the two superior vena cava. ${ }^{8,12}$ In most cases, persistent of LSVC results in the presence of both left and right superior vena cava. Diagnosis of LSVC is usually made as an incidental finding during cardiovascular imaging or surgery just like in the first case. In our second case, diagnosis of LSCV was found when we did venography after failed to advanced the wire into right atrium through RSVC due to the presence of trombus.

Presence of persistent LSVC can make transvenous lead implantation difficult. ${ }^{8}$ Any attemps should be made to define the anatomy of the superior caval system, especially if we met any difficulty using standart approach. Different techniques has been reported to place the lead to the right ventricle and minimize the risk of lead dislodgement. But sometimes these techniques prove unsuccessful and another site of venous access must be considered. ${ }^{13}$ In our first case, by using conventional methods (forming a loop in the right atrium using right atrial wall for support) 
lead could be placed in apical RV with no difficulty. In our second case we used a hand-shaped stillet and made a large loop for the lead to go to the apical of RV. We reshaped the wire and made a smaller loop. This manouvre was successful in directing the lead to RVOT. These cases showed that in persistent LSVC placement lead in apical and RVOT was visible and gave a good outcome.

Earlier experience had suggested a patient weight of $13-15 \mathrm{~kg}$ as the lower range for consideration of endocardial pacing.But some authors has performed uncomplicated implantation in smaller patient. 3,9 This based on consideration that epicardial approach is not a good choice in the early postoperative period even in small children because of significant tissue adhesions. ${ }^{9}$ So in our second case weighing 11 kilogram we used transvenous access for pacemaker implantation. We prefer to implanted single chamber pacemakers because of the higher risk of venous thrombosis in small children using dual chamber pacemaker. Active leads were used to prevent lead dislodgement, especially when we prefer RVOT pacing site. These cases has shown succesful permanent pacemaker implantation through persisten LSVC, one with dextrocardia, using some manouvres.

\section{References}

1. Batra AS, Wells WJ, Hinoki KW, Stanton RA, Silka MJ. Late recovery of atrioventricular conduction after pacemaker implantation for complete heart block associated with surgery for congenital heart disease. J Thorac Cardiovasc Surg. 2003;125(6):1291-1293

2. Nezafati MH, Abbasi M, Soltani G, Zirak. Early Postoperative Arrhythmia after Cardiac Surgery for Congenital Heart Diseases.
Iranian Heart J. 2008;9(3):53-58.

3. Chun T. Pacemaker and defibrillator therapy in pediatrics and congenital heart disease. Future Cardiol. 2008;4(5):469-479

4. Edwin F, Aniteye E, Tettey $M$ et al. Permanent complete heart block following surgical correction of congenital heart disease. Ghana Med J.44(3):109-114

5. Veneziani N, De Pasquale C, Ferlan G, et al. Pacemaker Implant in Dextrocardia with Right Superior Vena Cava and Persistence of Situs Inversus Viscerum. Progress in Biomedical Research 1999; 367-370

6. Fang Y, Jiang LC, and Chen M. Successful pacemaker implantation in a patient with dextrocardia situs inversus totalis. Europace (2009) 11, 1569, 699-700

7. Goyal SL, Lichestein E, Gupta PK, et al. Sick sinus syndrome requiring permanentpacemaker implantation in a patient with mirror-image dextrocardia. Chest 1976;69;558-561

8. Doshi AA, Cook SC, Hummel JD. Implantation of a BiVentricular Pacing System in the Setting of Dextrocardia with Situs Inversus Totalis. Indian Pacing and Electrophysiology Journal 2010, 10 (1): 58-61

9. Dalili M, Alizadeh A, Haghjoo M. Successful implantation of transvenous pacing system via persistent left superior vena cava and coronary sinus in small childre. Indian Pacing and Electrophysiology Journal 2010, 10 (12): 552-555

10. Palinkas A, Nagy E, Forster T. A case of absent right and persistent left superior vena cava. Cardiovascular Ultrasound 2006;4:6-9

11. Zerbe F, Bornakowski J, Sarnowski W. Pacemaker electrode implantation in patients with persistent left superior vena cava. Br Heart J 1992;67:65-66

12. Goyal SK, Punnam SR, verma G, Ruberg FL. Persistent left superior vena cava: a case report and review of literature. Cardiovascular Ultrasound 2008;6(50)

13. Srimannarayana J, Varma RS, Satheesh. Transvenous permanent pacemaker implantation through persistent left superior vena cava. Indian Heart J 2004;56:346-348 